\title{
Stochastic thermodynamics : the cost of time
}

\author{
Recommended papers :
}

Optimal protocols and optimal transport in stochastic thermodynamics, E. Aurell, C. Mejia-Monasterio, P. MuratoreGinanneschi. Phys. Rev. Lett. 106, 250601(1-4), (2011).

\section{Refined Second Law of Thermodynamics for Fast Random}

Processes. E. Aurell, K. Gawedzki, C. Mejia-Monasterio, R. Mohayaee, P. Muratore-Ginanneschi. Journal of Statistical Physics, 147, No. 3, 487505 (2012).

\section{Experimental verification of Landauer's principle linking} information and thermodynamics. Berut, A., Arakelyan, A., Petrosyan, A., Ciliberto, S., Dillenschneider R., et al.. Nature 483, 187-189 (2012).

Recommended with a commentary by M. Vergassola, Inst. Pasteur \& CNRS, Paris.

Classical thermodynamics has been generalized in recent years to the case of mesoand microscopic systems where fluctuations play a crucial role and contribute substantially to work and heat. A stochastic description of those quantities is thus needed and various exact relations, such as Jarzynski (1997), Crooks (1999), Hatano-Sasa (2001) and generalizations thereof, are now well-established and have been verified experimentally in a number of systems (see Bustamante et al. (2005) for an introduction and Ritort (2008) for a more advanced and detailed review).

In addition to the size of the system, another key factor brought into attention by outof-equilibrium relations is time, which was completely absent in thermodynamic adiabatic transformations. In stochastic thermodynamics (see, e.g., Seifert 2008) transformations can be driven at finite speed and some old and new questions become relevant and are worth consideration. Two such questions are the motivation and the main interest of the first two recommended papers: "What is the lower bound for the total entropy production or the average heat release in the process that interpolates between given states in a time interval of fixed length? "; "What is the dynamical protocol that leads to such a minimal total entropy production or heat release?». The last question refers to time-dependent protocols, e.g. mechanical forces, external fluxes, fixing concentrations of some chemical species in chemical reactions, etc., that are supposed to drive the system of interest.

The papers specifically consider an overdamped Langevin dynamics and establish a finite-time generalization of the classical Landauer principle (Landauer, 1961; Bennett, 1982). The latter states that the erasure of one bit of information during a computational process is coupled to heat exchanges with the thermal bath at temperature $T$ and that the release of an amount of heat equal to at least $k T$ (In2) (on average) is necessary. The classical example is a double well potential with a high 
barrier that gets slowly deformed so as to end up having a single minimum. What is the minimum amount of heat if (as it is always the case) we are not willing to wait forever and need to drive the system at finite (non-infinitesimal) speed ? This type of question was already addressed by Schmiedl \& Seifert (2007) for special cases where a solution for the driving protocol could be found analytically. The new papers demonstrate a general lower bound and, most importantly, establish a solid link with control theory (Bellmann, 1957; Kirk, 2004), the Monge-Kantorovich optimal mass transport (Villani, 2003) and the Burgers equation (Burgers, 1974). The final result is that the extra-cost generally decreases as the inverse of the time taken for the transformation whilst the constant of proportionality reflects the protocol that we employ to drive the system. The minimal value of this constant is achieved by driving the system with the protocol that solves the control problems mentioned above.

The third recommended paper uses an elegant and ingenious system of a single colloidal particle trapped in a modulated double-well potential to realize experimentally a model of a one-bit memory and then measures the heat exchanges. Long transformations indeed saturate Landauer bound. The extra-heat released for fast transformations is compatible with the finite-time lower bound derived in the second recommended paper and it will be of interest to experimentally drive the system with the optimal protocol to saturate the bound. Since all transformations in living organisms are obviously performed at finite speed, it seems fair to anticipate that the methods of stochastic thermodynamics will be important in the forthcoming years for quantitative investigations of biological systems.

\section{References}

C. Jarzynski, Nonequilibrium equality for free energy differences. Phys. Rev. Lett. 78, 2690 (1997).

G. E. Crooks, Entropy production fluctuation theorem and the nonequilibrium work relation for free energy differences. Phys. Rev. E 60, 2721 (1999).

T. Hatano and S. Sasa, Steady-State thermodynamics of Langevin systems Phys. Rev. Lett. 86, 3463 (2001).

C. Bustamante, J. Liphardt, and F. Ritort, The nonequilibrium thermodynamics of small systems. Phys. Today 58, No. 7, 43 (2005).

F. Ritort, Nonequilibrium fluctuations in small systems: from physics to biology, Advances in Chemical Physics, 137, 31-123 (2008).

Ch. H. Bennett, The Thermodynamics of Computation - a review, Int. J. Theor. Phys. 21 (1982), 905-940.

R. Landauer, Irreversibility and heat generation in the computing process, IBM Journal of Res. and Dev., 5:3 (1961), 183-191.

U. Seifert, Stochastic Thermodynamics : Principles and Perspectives. Eur. Phys. J. B 64, 423-431 (2008).

T. Schmiedl, U. Seifert, Optimal finite-time processes in stochastic thermodynamics, Phys. Rev. Lett. 98 (2007), 108301(1-4).

R. E. Bellman, Dynamic Programming, Princeton Univ. Press, Princeton (1957).

D.E. Kirk, Optimal Control Theory: An Introduction, Dover Publications (2004).

C. Villani, Topics in Optimal Transportation, Graduate Studies in Mathematics Vol.

38, American Mathematical Society, Providence R.I. 2003.

J. M. Burgers, The Nonlinear Diffusion Equation, D. Reidel, Dordrecht 1974. 